medRxiv preprint doi: https://doi.org/10.1101/2021.07.01.21259683; this version posted July 6, 2021. The copyright holder for this preprint (which was not certified by peer review) is the author/funder, who has granted medRxiv a license to display the preprint in perpetuity. It is made available under a CC-BY-NC-ND 4.0 International license .

\title{
Crowd Annotations Can Approximate Clinical Autism Impressions from Short Home Videos with Privacy Protections
}

\author{
Peter Washington, Stanford University \\ Emilie Leblanc, Stanford University \\ Kaitlyn Dunlap, Stanford University \\ Aaron Kline, Stanford University \\ Cezmi Mutlu, Stanford University \\ Brianna Chrisman, Stanford University \\ Nate Stockham, Stanford University \\ Kelley Paskov, Stanford University \\ Dennis P. Wall, Stanford University
}

\begin{abstract}
Artificial Intelligence (A.I.) solutions are increasingly considered for telemedicine. For these methods to adapt to the field of behavioral pediatrics, serving children and their families in home settings, it will be crucial to ensure the privacy of the child and parent subjects in the videos. To address this challenge in A.I. for healthcare, we explore the potential for global image transformations to provide privacy while preserving behavioral annotation quality. Crowd workers have previously been shown to reliably annotate behavioral features in unstructured home videos, allowing machine learning classifiers to detect autism using the annotations as input. We evaluate this method with videos altered via pixelation, dense optical flow, and Gaussian blurring. On a balanced test set of 30 videos of children with autism and 30 neurotypical controls, we find that the visual privacy alterations do not drastically alter any individual behavioral annotation at the item level. The AUROC on the evaluation set was $90.0 \%$ $+/-7.5 \%$ for the unaltered condition, $85.0 \%+/-9.0 \%$ for pixelation, $85.0 \%+/-9.0 \%$ for optical flow, and $83.3 \%+/-9.3 \%$ for blurring, demonstrating that an aggregation of small changes across multiple behavioral questions can collectively result in increased misdiagnosis rates. We also compare crowd answers against clinicians who provided the same annotations on the same videos and find that clinicians are more sensitive to autism-related symptoms. We also find that there is a linear correlation $(\mathrm{r}=0.75, \mathrm{p}<0.0001)$ between the mean Clinical Global Impression (CGI) score provided by professional clinicians and the corresponding classifier score emitted by the logistic regression classifier with crowd inputs, indicating that the classifier's output probability is a reliable estimate of clinical impression of autism from home videos. A significant correlation is maintained with privacy alterations, indicating that crowd annotations can approximate clinician-provided autism impression from home videos in a privacy-preserved manner.
\end{abstract}

\section{Introduction}

Artificial intelligence (A.I.) is increasingly being considered to help healthcare solutions scale. In order for such solutions to be deployed in clinical settings, several issues beyond simply achieving high performance must be addressed. In particular, the pipelines which host A.I. models must be seamless and intuitive for all users, and the system must garner maximal trust from all stakeholders. 
While A.I. approaches are needed for healthcare to achieve scale and consistency, current A.I.powered solutions to diagnostics within psychiatry and behavioral sciences are under-performant due to the complexity of the underling behaviors. Until A.I. has advanced to a point where it can seamlessly pass the Turing Test [39] and understand human social behavior, with all of its subtleties and nuances, to a degree that surpasses an average human's social acuity (i.e., to the level of a licensed clinician), it is unlikely that A.I. will replace any behavioral healthcare jobs. In the meantime, A.I. solutions which can augment the capabilities of non-expert humans may allow for scalable and accessible remote diagnostics while easing the burden of professional clinicians and the healthcare system to provide in person initial consultations.

Privacy rises to the forefront of patient concern when humans are incorporated into the diagnostic pipeline [56]. Many parents are uncomfortable with sharing raw videos of their children with strangers online even if the purpose of sharing is to help the parent's receive affordable and accessible diagnostic services [52]. However, human-in-the-loop techniques are pertinent given the current limitations of A.I., and privacy concerns must be directly addressed in any scalable solution where the humans in the loop are strangers (i.e., crowdsourced workers).

Here, we address these issues by studying an A.I.-augmented pipeline for detecting Autism Spectrum Disorder, or autism, from unstructured home videos. Autism is a developmental delay which is currently estimated to affect 1 in 40 children [27]. While access to care requires a formal diagnosis, access to diagnostic services is severely limited for many families, thus limiting potential care. Some evidence suggests that as much as $80 \%$ of families in the United States lack access to care [34], and underserved populations are disproportionately affected [17]. A.I. powered telemedical solutions therefore have the possibility to help these families, many of whom may otherwise lack access to traditional health services.

Crowd-powered telemedical diagnostic tools can provide parents with a risk score and an associated probability for a diagnosis, and prior works have repeatedly demonstrated the success of A.I. models to successfully detect autism using solely annotations provided by non-clinical human workers [5-8, 29, 37-38, 45, 47-48, 50-53, 57]. This family of solutions utilizes a distributed crowdsourced workforce to quickly annotate behavioral features displayed in videos recorded during parent-administered home autism therapy sessions using mobile digital health therapies [20-24] and wearable augmented reality solutions [2-4, 14-15, 26, 33, 40-43, 54-55].

In order for such solutions to truly scale for annotation by a large crowd workforce, the privacy of the patients must be preserved. This is important in general for healthcare applications, but it is especially critical when the patients in question are young children with a developmental delay and observed by a stranger in the privacy of their home. Our prior work has shown that applying light privacy-preserving modifications to videos, such as pitch shifting and covering the child's face with a virtual box, results in minimal degradation of the quality of crowdsourced annotations used for remote detection of autism-related behaviors [52]. However, such lightweight privacy protections may be insufficient to some patients, such as those patients who do not want the interior of their home exposed to strangers on the Internet. 
medRxiv preprint doi: https://doi.org/10.1101/2021.07.01.21259683; this version posted July 6, 2021. The copyright holder for this preprint (which was not certified by peer review) is the author/funder, who has granted medRxiv a license to display the preprint in perpetuity. It is made available under a CC-BY-NC-ND 4.0 International license .

Here, we explore the effect of standard visual privacy-preserving mechanisms. In particular, we apply pixelation, dense optical flow, and Gaussian blurring, which are either standard methods for protecting the privacy of human subjects in image and video datasets [35] or for representing visual features for activity recognition [25] while obfuscating personally identifiable visual features. We study the effect of these video transformations on annotation quality. On a balanced test set of 30 videos of children with autism and 30 neurotypical controls, we find that no visual privacy condition deviates from the unaltered condition by more than half of a categorical ordinal severity point out of 4 questions corresponding to behavior severity. We compare crowd responses to professional clinicians and find that the probability score emitted by the classifier is consistent with the clinicians' global impression from watching the same video. We also find that clinicians are more sensitive to autism-related symptoms than crowd workers.

\section{Methods}

\section{Balanced video dataset}

We leveraged a balanced video dataset of 30 children with autism and 30 controls without autism. Both groups were gender and aged matched: we posted 30 videos of male children (13 with autism and 17 neurotypical) and 30 videos of female children (17 with autism and 13 neurotypical). The mean age in the videos of children with autism was 3.49 years old $(\mathrm{SD}=1.58$ years old), and the mean age in the videos of children without autism was 3.41 years old $(\mathrm{SD}=1.39$ years old $)$.

\section{Logistic regression classifier for predicting autism}

We utilized a previously validated [37-38, 52] logistic regression classifier for predicting autism vs. not autism from the answers to the multiple-choice questions we asked crowd workers. The classifier [28, 30] was derived from the Autism Diagnostic Observation Schedule (ADOS) module 2 [31] and was trained on clinician filled scoresheets from the Boston Autism Consortium (AC), the Simons Simplex Collection v14 (SSC) [10], Autism Genetic Resource Exchange (AGRE) [11], National Database of Autism Research (NDAR) [16] and the Simons Variation in Individuals Project (SVIP) [36].

To generate confidence intervals, we performed 10,000 iterations for a bootstrapping procedure for each video. In each iteration, we sampled with replacement from the 60 videos used for evaluation and computed the accuracy, precision, recall / sensitivity, specificity, Area Under the Receiver Operating Characteristic (AUROC), and Area Under the Precision-Recall Curve (AUPRC) on the resulting video set.

\section{Privacy conditions}

We evaluated three privacy modifications applied to each video: (1) pixelation, (2) dense optical flow, and (3) Gaussian blur. Pixelation and Gaussian blurring are common methods for protecting the privacy of human subjects in image and video datasets [35]. Dense optical flow is a standard method for representing visual features for activity recognition [25] which happens to also preserve the privacy of the human subject in the video. To apply pixelation, we first resized 
medRxiv preprint doi: https://doi.org/10.1101/2021.07.01.21259683; this version posted July 6, 2021. The copyright holder for this preprint (which was not certified by peer review) is the author/funder, who has granted medRxiv a license to display the preprint in perpetuity. It is made available under a CC-BY-NC-ND 4.0 International license .

the input frames down to $32 \times 32$ pixels while applying bilinear interpolation. We then calculated the final pixelated frame by resizing the smaller frame back to the original frame size using bilinear interpolation. To calculate dense optical flow, we applied Farnebäck's algorithm for two-frame motion estimation based on polynomial expansion [9]. The image was colored through obtaining a 2-channel array with optical flow vectors, where the direction of the vectors corresponds to the hue of the image and the magnitude of the vectors corresponds to the value (lightness) of the image. To apply Gaussian blurring, we used a blurring kernel with 0.25 the width and height of the input image.

\section{Crowd annotation}

To recruit crowd workers, we posted a video rating task on the crowdsourcing website Microworkers.com [18]. In this video rating task, crowd workers are asked to answer a series of 13 multiple choice questions, with 4 answer choices each, about the child's behavior exhibited in each of 8 video videos ( 4 featuring a child with autism and 4 featuring a child without autism). We provided the categorical ordinal variables corresponding to each worker's answers into a pretrained binary logistic regression classifier for autism (see subsection Logistic regression classifier for predicting autism below for details about classifier training). 1,000 crowd workers completed the task and passed basic quality control checks for answer acceptance. Quality control measures included time spent on the annotation task and deviations in answers between videos [52]. We did not include workers who did not pass these basic quality control checks in the list of 1,000 evaluated workers.

We measured the classifier's prediction for all 8 filtering videos for all workers. We then measured the mean probability of the correct class (PCC) for each worker across all 8 videos, where the PCC is the classifier's output probability $p$ when the true class of a video is autism and $1-p$ otherwise. Out of the 1,000 workers evaluated, exactly 40 workers had a mean PCC at or above $80 \%$ when the mean PCC was rounded up. We recruited these 40 crowd workers to rate the primary video set of 60 videos used in this study.

Each of the 40 crowd workers were tasked with rating all 60 videos described in the subsection Balanced video dataset. We randomly split the 40 workers into 4 groups, and each group was assigned to one privacy condition per video. Therefore, no worker saw more than one version of each video. Furthermore, all workers saw exactly 15 unaltered videos, 15 videos with pixelation, 15 videos with dense optical flow, and 15 videos with Gaussian blurring. This ensured that no privacy condition was biased by any crowd worker annotation biases.

\section{Clinician annotation}

We recruited 19 clinicians to rate the same balanced video dataset and provide the same categorical ordinal video-wide annotations as the crowd workers. All clinicians were licensed professionals who provide diagnoses of autism as part of their job duties. All clinicians were asked an additional question which crowd workers were not asked: "Do you think the child has autism?" The answer choices were:

- No, I am confident the child does not have autism (0) 
- No, but I am unsure (1)

- Yes, but I am unsure (2)

- Yes, I am confident the child has autism (3)

All 60 videos received at least 1 complete rating by at least 1 clinician. Some videos were rated by more than 1 clinician, in which case we recorded the mean of the clinician answers for that video. For the "Do you think the child has autism?" question, we coded the responses from 0 to 3 as shown above.

Clinicians were also asked to provide a Clinical Global Impression (CGI) [12] rating for the children in the videos. The CGI scale measures the "severity of illness" between 1 ("normal, not at all ill") to 7 ("among the most extremely ill patients") and is designed to allow clinicians to provide a global impression without providing a formal diagnosis.

\section{Item-level analysis}

For each question we asked crowd workers, we measured the mean absolute deviation of the mean answer for each privacy condition from the mean answer for the baseline condition. This difference provides a measure of the privacy condition's effect on annotation quality. We hypothesized that some questions would be more susceptible to alteration with certain privacy conditions applied.

\section{Results}

All procedures performed in studies involving human participants were in accordance with the ethical standards of the institution (approved by the Stanford University Institutional Review Board) and with the 1964 Helsinki declaration and its later amendments or comparable ethical standards.

\section{Performance of clinicians and crowd workers}

Out of the 30 videos of children with autism, clinicians rated 8 videos confidently (with a mean autism rating above 2.5 out of 3.0). By contrast, clinicians rated 18 of the videos of neurotypical children confidently (with a mean autism rating below 0.5 out of 3.0 ). All 8 confidently rated videos were of children with autism, while 16 of the 18 neurotypical children were correctly identified (only 2 were actually diagnosed with autism). This suggests that clinicians observing remote videos of children are cautious about calling an autism diagnosis, but when they do guess a diagnosis, the child is very likely to actually have autism.

The clinician's classifier correctly identified 28 of the 30 autism cases while only correctly identifying 14 of the 30 neurotypical cases. By contrast, the crowd's classifier correctly identified 25 of the 30 autism cases and 27 of the 30 neurotypical cases. This suggests that clinicians are more sensitive to autism-related symptoms than crowd workers, thus resulting in a higher frequency of autism diagnosis by the binary classifiers. 
There is a clear linear correlation $(\mathrm{r}=0.75, \mathrm{p}<0.001)$ between the mean Clinical Global Impression (CGI) score provided by professional clinicians for each video and the corresponding classifier score emitted by the logistic regression classifier with crowd inputs (Figure 1). This suggests that crowd responses in conjunction with machine learning algorithms can approximate clinician intuition, and the classifier's output can be interpreted as a fairly reliable approximation of clinical global impressions of autism.

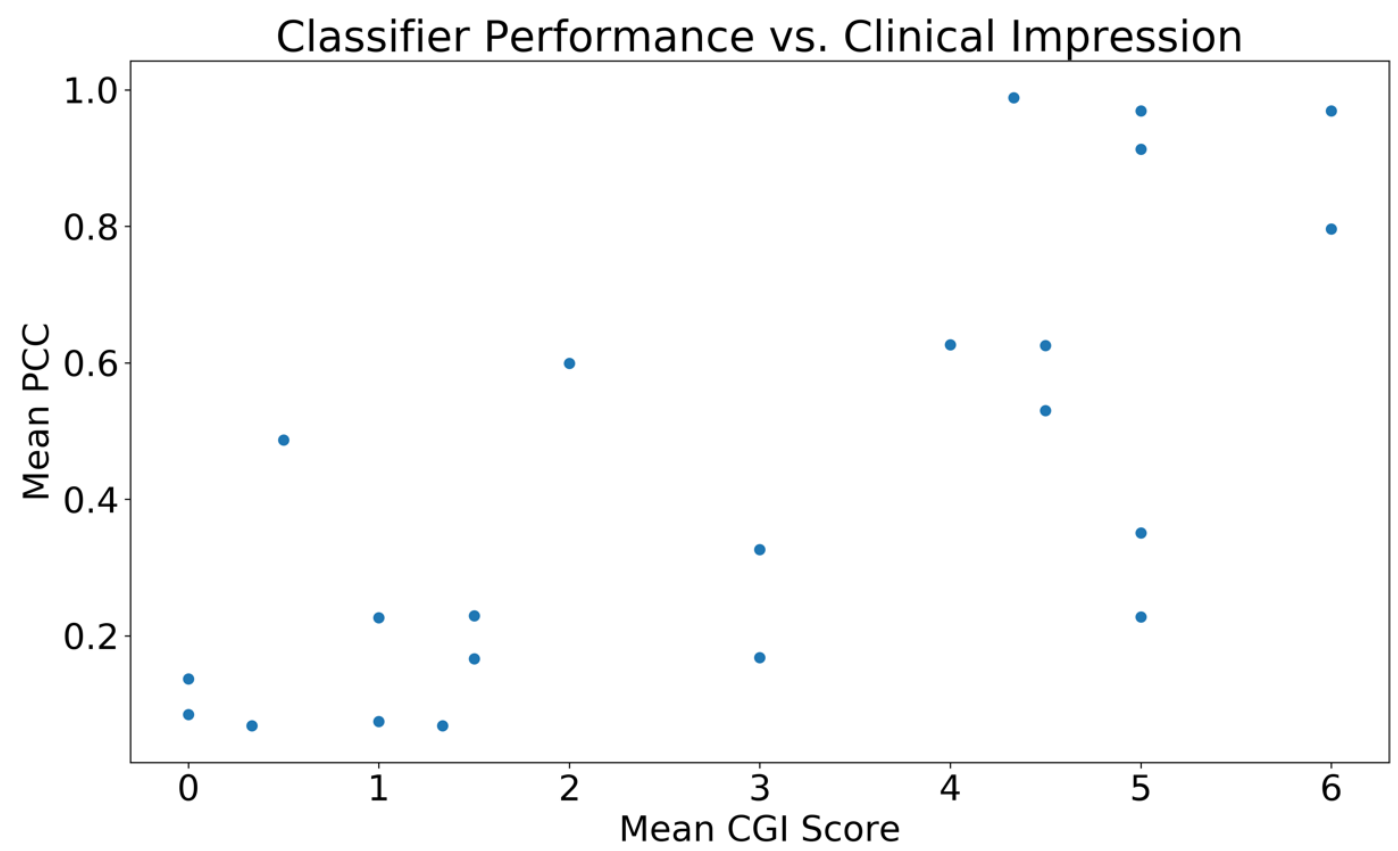

Figure 1. There is a clear linear correlation $(r=0.75, \mathrm{p}<0.001)$ between the mean Clinical Global Impression (CGI) score provided by professional clinicians for each video and the corresponding classifier score emitted by the logistic regression classifier with crowd inputs.

Of the 60 unaltered videos, the mean performance metrics were $90.0 \%+/-7.5 \%$ accuracy, $92.9 \%$ $+/-8.9 \%$ precision, $86.7 \%+/-11.8 \%$ recall (sensitivity), and $93.3 \%+/-8.6 \%$ specificity. The mean AUROC was $90.0 \%+/-7.5 \%$ and the mean AUPRC was $93.1 \%+/-6.2 \%$.

\section{Effect of privacy conditions}

The linear correlation between the mean Clinical Global Impression (CGI) score provided by professional clinicians for each video and the corresponding classifier score emitted by the logistic regression classifier with crowd inputs is maintained with privacy-preserving video modifications. The correlation is weaker for Gaussian blurring $(\mathrm{r}=0.64, \mathrm{p}=0.001$ for Gaussian blurring) than for dense optical flow and pixelation ( $\mathrm{r}=0.71, \mathrm{p}=0.0002$ for pixelation for both).

With pixelation, the mean performance metrics were $85.0 \%+/-9.2 \%$ accuracy, $88.9 \%+/$ $12.1 \%$ precision, $80.0 \%+/-14.4 \%$ recall (sensitivity), and $90.0 \%+/-10.9 \%$ specificity. The mean AUROC was $85.0 \%+/-9.0 \%$ and the mean AUPRC was $89.4 \%+/-7.9 \%$. 
With dense optical flow, the mean performance metrics were $85.0 \%+/-9.2 \%$ accuracy, $81.8 \%$ $+/-13.1 \%$ precision, $90.0 \%+/-10.9 \%$ recall (sensitivity), and $80.0 \%+/-14.4 \%$ specificity. The mean AUROC was $85.0 \%+/-9.0 \%$ and the mean AUPRC was $88.4 \%+/-7.9 \%$.

With Gaussian blurring, the mean performance metrics were $83.3 \%+/-9.2 \%$ accuracy, $83.3 \%$ $+/-13.7 \%$ precision, $83.3 \%+/-13.7 \%$ recall (sensitivity), and $83.3 \%+/-13.7 \%$ specificity. The mean AUROC was $83.3 \%+/-9.3 \%$ and the mean AUPRC was $87.5 \%+/-8.5 \%$.

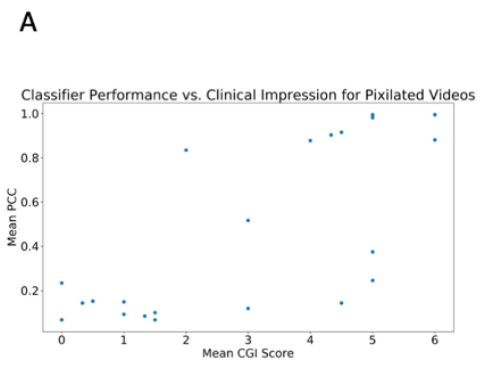

\section{B}

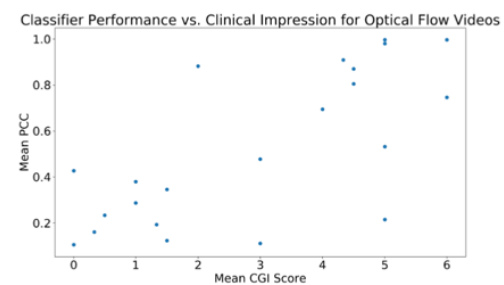

C

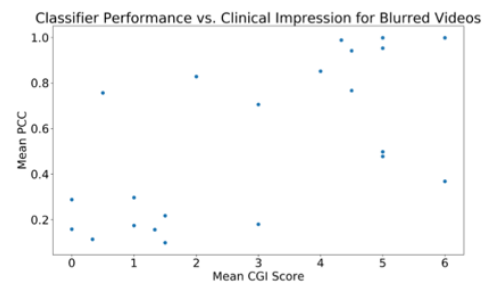

Figure 2. The linear correlation between the mean Clinical Global Impression (CGI) score provided by professional clinicians for each video and the corresponding classifier score emitted

by the logistic regression classifier with crowd inputs is maintained with privacy-preserving video modifications. The correlation is weaker for Gaussian blurring $(r=0.64, p=0.001$ for Gaussian blurring) than for dense optical flow and pixelation ( $\mathrm{r}=0.71, \mathrm{p}=0.0002$ for both).

\section{Item-level analysis}

Table 1 displays the mean absolute deviation of the mean answer for each privacy condition from the mean answer for the baseline condition. This difference provides a measure of the privacy condition's effect on annotation.

\begin{tabular}{|c|c|c|c|}
\hline & $\begin{array}{c}\text { Mean Deviation } \\
\text { for Pixelation }\end{array}$ & $\begin{array}{c}\text { Mean Deviation } \\
\text { for Dense } \\
\text { Optical Flow }\end{array}$ & $\begin{array}{c}\text { Mean Deviation } \\
\text { for Gaussian } \\
\text { Blurring }\end{array}$ \\
\hline $\begin{array}{c}\text { Abnormal } \\
\text { Speech }\end{array}$ & 0.28 & 0.32 & 0.29 \\
\hline Echolalia & 0.39 & 0.45 & 0.47 \\
\hline $\begin{array}{c}\text { Repetitive or } \\
\text { Odd Language }\end{array}$ & 0.25 & 0.30 & 0.26 \\
\hline $\begin{array}{c}\text { Expressive } \\
\text { Language and } \\
\text { Conversation }\end{array}$ & 0.29 & 0.41 & 0.33 \\
\hline Eye Contact & 0.29 & 0.37 & 0.33 \\
\hline $\begin{array}{c}\text { Facial } \\
\text { Expressiveness }\end{array}$ & 0.25 & 0.32 & 0.29 \\
\hline $\begin{array}{c}\text { Social } \\
\text { Interaction } \\
\text { Initiation }\end{array}$ & 0.26 & 0.30 & 0.31 \\
\hline
\end{tabular}


medRxiv preprint doi: https://doi.org/10.1101/2021.07.01.21259683; this version posted July 6, 2021. The copyright holder for this preprint (which was not certified by peer review) is the author/funder, who has granted medRxiv a license to display the preprint in perpetuity. It is made available under a CC-BY-NC-ND 4.0 International license .

\begin{tabular}{|c|c|c|c|}
\hline $\begin{array}{c}\text { Shares } \\
\text { Excitement }\end{array}$ & 0.34 & 0.32 & 0.37 \\
\hline $\begin{array}{c}\text { Aggressive } \\
\text { Behavior }\end{array}$ & 0.09 & 0.12 & 0.12 \\
\hline
\end{tabular}

Table 1. The mean absolute deviation for each privacy condition from the baseline condition answers for the behaviors used in the autism classifiers. This difference provides a measure of the privacy condition's effect on annotation quality.

We found that pixelation resulted in smaller deviations from the unmodified video condition compared to dense optical flow and Gaussian blurring. In all but one behavioral annotation (for sharing excitement), the mean deviation for pixelation was less than for the other two privacy conditions. Dense optical flow, which provides maximal privacy, did not have a discernable difference from Gaussian blurring (private but less so), providing support for the use of dense optical flow in translational settings.

The annotation with the lowest deviation across all conditions was for displaying aggressive behavior. This makes intuitive sense and serves as a sanity check, as no aggressive behavior was displayed in any of the videos we presented.

None of the 9 behaviors used for the classifiers contained a mean deviation above 0.5 . This means that the mean deviation is less than one half of the distance between one categorical ordinal variable representing symptom severity and the variable indicating one severity level higher (all questions contained 4 multiple choice options). We note that while these deviations are consistently small, the aggregation of these deviations results in more misclassifications (see Results: Effect of privacy conditions).

\section{Discussion and Conclusion}

We explored the potential for global image transformations to provide privacy for video subjects while still preserving behavioral annotation quality. While no individual question was drastically degraded, some behavioral annotations were degraded more than others. Pixelation consistently resulted in less drastic degradations than blurring and optical flow. We also found that the classifier's predictions from the crowd's annotation of the unaltered videos were strongly correlated with clinician global impressions. A slightly less strong correlation persisted even after all privacy modifications we tested, providing evidence that the classifier's output can potentially be considered as an estimation of clinical global autism impression scores even when annotations are provided for a privacy-preserved video stream.

There are several limitations to the current study. While the configuration of questions we asked workers and clinicians resulted in worse performance by the clinicians' annotations, this could have been due to over-sensitivity by the classifier rather than anything the clinicians did wrong. We therefore do not make any claims about the performance of clinicians as compared to crowd workers. An interesting limitation is that the definition of autism tends to shift over time with evolving DSM criteria and clinical practices [32]. Because clinicians were providing annotations several years after the videos of children were recorded, it is possible that the children who did 
not qualify for a diagnosis at the time the videos were recorded would qualify for a diagnosis by the time the clinicians reviewed the videos.

There are several interesting avenues of future work. The annotations provided by crowd workers can potentially be used to train computer vision classifiers detecting behaviors relevant to autism detection such as emotion evocation $[15,19,44]$, hand or head stimming [46], and abnormal eye contact. While humans are worse at detecting certain behavioral patterns from videos when privacy mechanisms are applied, it is possible that convolutional neural networks can more easily detect these features by learning subtle and nonlinear feature maps beyond human comprehension. As machine learning algorithms improve and relevant databases become more plentiful, the possibility of removing humans from the remote detection pipeline seems increasingly inevitable.

Future work should ensure that all methods work for all stakeholders and such methods should therefore be evaluated across races and ethnicities to ensure fair and unbiased A.I. While some machine learning methods may help account for biased datasets, no technique matches the benefit of using balanced data. Fair and balanced healthcare A.I. initiatives must explicitly recruit participants in equal numbers across all demographics served to allow for equitable services for all.

\section{Acknowledgements}

We thank all crowd workers who participated in the study. We also thank the clinicians who provided annotations for all study videos.

These studies were supported by awards to DPW by the National Institutes of Health (1R21HD091500-01 and 1R01EB025025-01). Additionally, we acknowledge the support of grants to DPW from The Hartwell Foundation, the David and Lucile Packard Foundation Special Projects Grant, Beckman Center for Molecular and Genetic Medicine, Coulter Endowment Translational Research Grant, Berry Fellowship, Spectrum Pilot Program, Stanford's Precision Health and Integrated Diagnostics Center (PHIND), Wu Tsai Neurosciences Institute Neuroscience: Translate Program, Spark Program in Translational Research, Stanford's Institute of Human Centered Artificial Intelligence, the Weston Havens Foundation, as well as philanthropic support from Peter Sullivan. PW would like to acknowledge support from the Stanford Interdisciplinary Graduate Fellowship (SIGF).

\section{References}

1. Adler, B. Andrew, Noha F. Minshawi, and Craig A. Erickson. "Evolution of autism: From Kanner to the DSM-V." In Handbook of Early Intervention for Autism Spectrum Disorders, pp. 3-19. Springer, New York, NY, 2014.

2. Daniels, Jena, Jessey Schwartz, Nick Haber, Catalin Voss, Aaron Kline, Azar Fazel, Peter Washington et al. "5.13 Design and efficacy of a wearable device for social affective learning in children with autism." Journal of the American Academy of Child \& Adolescent Psychiatry 56, no. 10 (2017): S257.

3. Daniels, Jena, Nick Haber, Catalin Voss, Jessey Schwartz, Serena Tamura, Azar Fazel, Aaron Kline et al. "Feasibility testing of a wearable behavioral aid for social learning in children with autism." Applied clinical informatics 9, no. 1 (2018): 129.

4. Daniels, Jena, Jessey N. Schwartz, Catalin Voss, Nick Haber, Azar Fazel, Aaron Kline, Peter Washington, Carl Feinstein, Terry Winograd, and Dennis P. Wall. "Exploratory study examining 
the at-home feasibility of a wearable tool for social-affective learning in children with autism." NPJ digital medicine 1, no. 1 (2018): 1-10.

5. Duda, Marlena, Jena Daniels, and Dennis P. Wall. "Clinical evaluation of a novel and mobile autism risk assessment." Journal of autism and developmental disorders 46, no. 6 (2016): 19531961.

6. Duda, M., N. Haber, J. Daniels, and D. P. Wall. "Crowdsourced validation of a machine-learning classification system for autism and ADHD." Translational psychiatry 7, no. 5 (2017): e1133e1133.

7. Duda, M., J. A. Kosmicki, and D. P. Wall. "Testing the accuracy of an observation-based classifier for rapid detection of autism risk." Translational psychiatry 5, no. 4 (2015): e556-e556.

8. Duda, M., R. Ma, N. Haber, and D. P. Wall. "Use of machine learning for behavioral distinction of autism and ADHD." Translational psychiatry 6, no. 2 (2016): e732-e732.

9. Farnebäck, Gunnar. "Two-frame motion estimation based on polynomial expansion." In Scandinavian conference on Image analysis, pp. 363-370. Springer, Berlin, Heidelberg, 2003.

10. Fischbach, Gerald D., and Catherine Lord. "The Simons Simplex Collection: a resource for identification of autism genetic risk factors." Neuron 68, no. 2 (2010): 192-195.

11. Geschwind, Daniel H., Janice Sowinski, Catherine Lord, Portia Iversen, Jonathan Shestack, Patrick Jones, Lee Ducat, and Sarah J. Spence. "The autism genetic resource exchange: a resource for the study of autism and related neuropsychiatric conditions." The American Journal of Human Genetics 69, no. 2 (2001): 463-466.

12. Guy, William. ECDEU assessment manual for psychopharmacology. US Department of Health, Education, and Welfare, Public Health Service, Alcohol, Drug Abuse, and Mental Health Administration, National Institute of Mental Health, Psychopharmacology Research Branch, Division of Extramural Research Programs, 1976.

13. Haber, Nick, Catalin Voss, and Dennis Wall. "Making emotions transparent: Google Glass helps autistic kids understand facial expressions through augmented-reaiity therapy." IEEE Spectrum 57, no. 4 (2020): 46-52.

14. Haber, Nick, Catalin Voss, Jena Daniels, Peter Washington, Azar Fazel, Aaron Kline, Titas De, Terry Winograd, Carl Feinstein, and Dennis P. Wall. "A Wearable Social Interaction Aid for Children with Autism." arXiv preprint arXiv:2004.14281 (2020).

15. Haber, Nick, Catalin Voss, Azar Fazel, Terry Winograd, and Dennis P. Wall. "A practical approach to real-time neutral feature subtraction for facial expression recognition." In 2016 IEEE Winter Conference on Applications of Computer Vision (WACV), pp. 1-9. IEEE, 2016.

16. Hall, Dan, Michael F. Huerta, Matthew J. McAuliffe, and Gregory K. Farber. "Sharing heterogeneous data: the national database for autism research." Neuroinformatics 10, no. 4 (2012): 331-339.

17. Howlin, Patricia, and Anna Moore. "Diagnosis in autism: A survey of over 1200 patients in the UK." autism 1, no. 2 (1997): 135-162.

18. Hirth, Matthias, Tobias Hoßfeld, and Phuoc Tran-Gia. "Anatomy of a crowdsourcing platformusing the example of microworkers. com." In 2011 Fifth international conference on innovative mobile and internet services in ubiquitous computing, pp. 322-329. IEEE, 2011.

19. Hou, Cathy, Haik Kalantarian, Peter Washington, Kaiti Dunlap, and Dennis Wall. "Development and Validation of a Facial Emotion Classifier for Applications in the Treatment of Autism Spectrum Disorder."

20. Kalantarian, Haik, Khaled Jedoui, Kaitlyn Dunlap, Jessey Schwartz, Peter Washington, Arman Husic, Qandeel Tariq, Michael Ning, Aaron Kline, and Dennis Paul Wall. "The performance of emotion classifiers for children with parent-reported autism: quantitative feasibility study." JMIR Mental Health 7, no. 4 (2020): e13174.

21. Kalantarian, Haik, Khaled Jedoui, Peter Washington, Qandeel Tariq, Kaiti Dunlap, Jessey Schwartz, and Dennis P. Wall. "Labeling images with facial emotion and the potential for pediatric healthcare." Artificial intelligence in medicine 98 (2019): 77-86.

22. Kalantarian, Haik, Khaled Jedoui, Peter Washington, and Dennis P. Wall. "A mobile game for automatic emotion-labeling of images." IEEE transactions on games 12, no. 2 (2018): 213-218. 
medRxiv preprint doi: https://doi.org/10.1101/2021.07.01.21259683; this version posted July 6, 2021. The copyright holder for this preprint (which was not certified by peer review) is the author/funder, who has granted medRxiv a license to display the preprint in perpetuity. It is made available under a CC-BY-NC-ND 4.0 International license .

23. Kalantarian, Haik, Peter Washington, Jessey Schwartz, Jena Daniels, Nick Haber, and Dennis P. Wall. "Guess what?." Journal of healthcare informatics research 3, no. 1 (2019): 43-66.

24. Kalantarian, Haik, Peter Washington, Jessey Schwartz, Jena Daniels, Nick Haber, and Dennis Wall. "A gamified mobile system for crowdsourcing video for autism research." In 2018 IEEE international conference on healthcare informatics (ICHI), pp. 350-352. IEEE, 2018.

25. Ke, Shian-Ru, Hoang Le Uyen Thuc, Yong-Jin Lee, Jenq-Neng Hwang, Jang-Hee Yoo, and Kyoung-Ho Choi. "A review on video-based human activity recognition." Computers 2, no. 2 (2013): 88-131.

26. Kline, Aaron, Catalin Voss, Peter Washington, Nick Haber, Hessey Schwartz, Qandeel Tariq, Terry Winograd, Carl Feinstein, and Dennis P. Wall. "Superpower glass." GetMobile: Mobile Computing and Communications 23, no. 2 (2019): 35-38.

27. Kogan, Michael D., Catherine J. Vladutiu, Laura A. Schieve, Reem M. Ghandour, Stephen J. Blumberg, Benjamin Zablotsky, James M. Perrin et al. "The prevalence of parent-reported autism spectrum disorder among US children." Pediatrics 142, no. 6 (2018).

28. Kosmicki, J. A., V. Sochat, M. Duda, and D. P. Wall. "Searching for a minimal set of behaviors for autism detection through feature selection-based machine learning." Translational psychiatry 5 , no. 2 (2015): e514-e514.

29. Leblanc, Emilie, Peter Washington, Maya Varma, Kaitlyn Dunlap, Yordan Penev, Aaron Kline, and Dennis P. Wall. "Feature replacement methods enable reliable home video analysis for machine learning detection of autism." Scientific reports 10, no. 1 (2020): 1-11.

30. Levy, Sebastien, Marlena Duda, Nick Haber, and Dennis P. Wall. "Sparsifying machine learning models identify stable subsets of predictive features for behavioral detection of autism." Molecular autism 8, no. 1 (2017): 1-17.

31. Lord, Catherine, Susan Risi, Linda Lambrecht, Edwin H. Cook, Bennett L. Leventhal, Pamela C. DiLavore, Andrew Pickles, and Michael Rutter. "The Autism Diagnostic Observation ScheduleGeneric: A standard measure of social and communication deficits associated with the spectrum of autism." Journal of autism and developmental disorders 30, no. 3 (2000): 205-223.

32. Mintz, Mark. "Evolution in the understanding of autism spectrum disorder: historical perspective." The Indian Journal of Pediatrics 84, no. 1 (2017): 44-52.

33. Nag, Anish, Nick Haber, Catalin Voss, Serena Tamura, Jena Daniels, Jeffrey Ma, Bryan Chiang et al. "Toward continuous social phenotyping: analyzing gaze patterns in an emotion recognition task for children with autism through wearable smart glasses." Journal of medical Internet research 22, no. 4 (2020): e13810.

34. Ning, Michael, Jena Daniels, Jessey Schwartz, Kaitlyn Dunlap, Peter Washington, Haik Kalantarian, Michael Du, and Dennis P. Wall. "Identification and quantification of gaps in access to autism resources in the United States: an infodemiological study." Journal of medical Internet research 21, no. 7 (2019): e13094.

35. Padilla-López, José Ramón, Alexandros Andre Chaaraoui, and Francisco Flórez-Revuelta. "Visual privacy protection methods: A survey." Expert Systems with Applications 42, no. 9 (2015): 4177-4195.

36. Simons VIP Consortium. "Simons Variation in Individuals Project (Simons VIP): a genetics-first approach to studying autism spectrum and related neurodevelopmental disorders." Neuron 73, no. 6 (2012): 1063-1067.

37. Tariq, Qandeel, Jena Daniels, Jessey Nicole Schwartz, Peter Washington, Haik Kalantarian, and Dennis Paul Wall. "Mobile detection of autism through machine learning on home video: A development and prospective validation study." PLoS medicine 15, no. 11 (2018): e1002705.

38. Tariq, Qandeel, Scott Lanyon Fleming, Jessey Nicole Schwartz, Kaitlyn Dunlap, Conor Corbin, Peter Washington, Haik Kalantarian, Naila Z. Khan, Gary L. Darmstadt, and Dennis Paul Wall. "Detecting developmental delay and autism through machine learning models using home videos of Bangladeshi children: Development and validation study." Journal of medical Internet research 21, no. 4 (2019): e13822.

39. Turing, Alan M. "Computing machinery and intelligence." In Parsing the turing test, pp. 23-65. Springer, Dordrecht, 2009. 
medRxiv preprint doi: https://doi.org/10.1101/2021.07.01.21259683; this version posted July 6, 2021. The copyright holder for this preprint (which was not certified by peer review) is the author/funder, who has granted medRxiv a license to display the preprint in perpetuity. It is made available under a CC-BY-NC-ND 4.0 International license .

40. Voss, Catalin, Nick Haber, and Dennis P. Wall. "The Potential for Machine Learning-Based Wearables to Improve Socialization in Teenagers and Adults With Autism Spectrum DisorderReply." JAMA pediatrics 173, no. 11 (2019): 1106-1106.

41. Voss, Catalin, Nick Haber, Peter Washington, Aaron Kline, Beth McCarthy, Jena Daniels, Azar Fazel et al. "Designing a Holistic At-Home Learning Aid for Autism." arXiv preprint arXiv:2002.04263 (2020).

42. Voss, Catalin, Jessey Schwartz, Jena Daniels, Aaron Kline, Nick Haber, Peter Washington, Qandeel Tariq et al. "Effect of wearable digital intervention for improving socialization in children with autism spectrum disorder: a randomized clinical trial." JAMA pediatrics 173, no. 5 (2019): 446-454.

43. Voss, Catalin, Peter Washington, Nick Haber, Aaron Kline, Jena Daniels, Azar Fazel, Titas De et al. "Superpower glass: delivering unobtrusive real-time social cues in wearable systems." In Proceedings of the 2016 ACM International Joint Conference on Pervasive and Ubiquitous Computing: Adjunct, pp. 1218-1226. 2016.

44. Washington, Peter, Haik Kalantarian, Jack Kent, Arman Husic, Aaron Kline, Emilie Leblanc, Cathy Hou et al. "Training an Emotion Detection Classifier using Frames from a Mobile Therapeutic Game for Children with Developmental Disorders." arXiv preprint arXiv:2012.08678 (2020).

45. Washington, Peter, Haik Kalantarian, Qandeel Tariq, Jessey Schwartz, Kaitlyn Dunlap, Brianna Chrisman, Maya Varma et al. "Validity of online screening for autism: crowdsourcing study comparing paid and unpaid diagnostic tasks." Journal of medical Internet research 21, no. 5 (2019): e13668.

46. Washington, Peter, Aaron Kline, Onur Cezmi Mutlu, Emilie Leblanc, Cathy Hou, Nate Stockham, Kelley Paskov, Brianna Chrisman, and Dennis P. Wall. "Activity Recognition with Moving Cameras and Few Training Examples: Applications for Detection of Autism-Related Headbanging." arXiv preprint arXiv:2101.03478 (2021).

47. Washington, Peter, Emilie Leblanc, Kaitlyn Dunlap, Yordan Penev, Aaron Kline, Kelley Paskov, Min Woo Sun et al. "Precision Telemedicine through Crowdsourced Machine Learning: Testing Variability of Crowd Workers for Video-Based Autism Feature Recognition." Journal of personalized medicine 10, no. 3 (2020): 86.

48. Washington, Peter, Emilie Leblanc, Kaitlyn Dunlap, Yordan Penev, Maya Varma, Jae-Yoon Jung, Brianna Chrisman et al. "Selection of trustworthy crowd workers for telemedical diagnosis of pediatric autism spectrum disorder." In BIOCOMPUTING 2021: Proceedings of the Pacific Symposium, pp. 14-25. 2020.

49. Washington, Peter, Onur Cezmi Mutlu, Emilie Leblanc, Aaron Kline, Cathy Hou, Brianna Chrisman, Nate Stockham et al. "Using Crowdsourcing to Train Facial Emotion Machine Learning Models with Ambiguous Labels." arXiv preprint arXiv:2101.03477 (2021).

50. Washington, Peter, Natalie Park, Parishkrita Srivastava, Catalin Voss, Aaron Kline, Maya Varma, Qandeel Tariq et al. "Data-driven diagnostics and the potential of mobile artificial intelligence for digital therapeutic phenotyping in computational psychiatry." Biological Psychiatry: Cognitive Neuroscience and Neuroimaging (2019).

51. Washington, Peter, Kelley Marie Paskov, Haik Kalantarian, Nathaniel Stockham, Catalin Voss, Aaron Kline, Ritik Patnaik et al. "Feature selection and dimension reduction of social autism data." In PACIFIC SYMPOSIUM ON BIOCOMPUTING 2020, pp. 707-718. 2019.

52. Washington, Peter, Qandeel Tariq, Emilie Leblanc, Brianna Chrisman, Kaitlyn Dunlap, Aaron Kline, Haik Kalantarian et al. "Crowdsourced privacy-preserved feature tagging of short home videos for machine learning ASD detection." Scientific reports 11, no. 1 (2021): 1-11.

53. Washington, Peter, Qandeel Tariq, Emilie Leblanc, Brianna Chrisman, Kaitlyn Dunlap, Aaron Kline, Haik Kalantarian et al. "Crowdsourced feature tagging for scalable and privacy-preserved autism diagnosis." medRxiv (2020).

54. Washington, Peter, Catalin Voss, Aaron Kline, Nick Haber, Jena Daniels, Azar Fazel, Titas De, Carl Feinstein, Terry Winograd, and Dennis Wall. "Superpowerglass: a wearable aid for the at- 
medRxiv preprint doi: https://doi.org/10.1101/2021.07.01.21259683; this version posted July 6, 2021. The copyright holder for this preprint (which was not certified by peer review) is the author/funder, who has granted medRxiv a license to display the preprint in perpetuity. It is made available under a CC-BY-NC-ND 4.0 International license .

home therapy of children with autism." Proceedings of the ACM on interactive, mobile, wearable and ubiquitous technologies 1, no. 3 (2017): 1-22.

55. Washington, Peter, Catalin Voss, Nick Haber, Serena Tanaka, Jena Daniels, Carl Feinstein, Terry Winograd, and Dennis Wall. "A wearable social interaction aid for children with autism." In Proceedings of the $2016 \mathrm{CHI}$ Conference Extended Abstracts on Human Factors in Computing Systems, pp. 2348-2354. 2016.

56. Washington, Peter, Serena Yeung, Bethany Percha, Nicholas Tatonetti, Jan Liphardt, and Dennis P. Wall. "Achieving trustworthy biomedical data solutions." In BIOCOMPUTING 2021:

Proceedings of the Pacific Symposium, pp. 1-13. 2020.

57. Wall, Dennis Paul, J. Kosmicki, T. F. Deluca, E. Harstad, and Vincent Alfred Fusaro. "Use of machine learning to shorten observation-based screening and diagnosis of autism." Translational psychiatry 2, no. 4 (2012): e100-e100. 